\title{
Commentary
}

\section{Hypotheses of Cancer Weakening and Origin}

\author{
John Cheung Yuen CHAN ${ }^{凶}$ \\ 33 Magnus St, Nelson Bay NSW 2315 Australia
}

$\square$ Corresponding author: Mobile: +61 (0) 428688 800, Email: chantrading@bigpond.com

() 2015 Ivyspring International Publisher. Reproduction is permitted for personal, noncommercial use, provided that the article is in whole, unmodified, and properly cited. See http://ivyspring.com/terms for terms and conditions.

Received: 2014.10.26; Accepted: 2015.01.26; Published: 2015.03.09

\begin{abstract}
Approximately 2.7 billion years ago, cyanobacteria began producing oxygen by photosynthesis. Any free oxygen they produced was chemically captured by dissolved iron or organic matter. There was no ozone layer to protect living species against the radiation from space. Eukaryotic cells lived in water, under hypoxic environments, and metabolized glucose by fermentation. The Great Oxygenation Event (GOE) describes the point when oxygen sinks became saturated. This massive oxygenation of the Earth occurred approximately half a billion years ago. Species that evolved after the GOE are characterized by aerobic metabolism. Mammals evolved approximately a few hundred million years ago, with the ancient eukaryotic genes deeply embedded in their genome.

Many genes have been exchanged by horizontal gene transfer (HGT) throughout the history of cellular evolution. Mammals have been invaded by viruses, and while viral genetic relics are embedded in mammalian junk genes, not all junk genes are genetic relics of viruses. These viral relics have been inactivated through evolution and have little impact on mammalian life. However, there is evidence to suggest that these viral genetic relics are linked to cancer.

This hypothesis states that cancer develops when cell reproduction becomes defective because of the active involvement of viral genes, in a process similar to genetic engineering. Cancer cells are amalgamations of genetically modified organisms (GMOs). There are two main groups in cancer development. One group of cells arises by genetic engineering of a viral genetic relic, such as endogenous retroviruses (ERVs), which evolved after oxygenation of the atmosphere. This group is referred to here as genetically modified organisms from viral genes (GMOV). GMOVs may be inhibited by anticancer drugs. The second group arises by engineering of the genes of ancient eukaryotes, which existed prior to the oxygenation of the Earth. This second group is referred to as genetically modified organisms from ancient eukaryotic genes (GMOE). The GMOE group lives in hypoxic environments and metabolizes glucose by fermentation. GMOEs represent advanced cancer, which proliferate aggressively and are resistant to DNA damage.

It has been demonstrated that as an ERV becomes more prevalent in a mammalian genome, the possibility that the mammal will develop cancer increases. The hypothesis also states that most cancers have their origins in GMOV by the incorporation of viral genes from junk genes. As the cancer progresses, further subgroups of cancer GMOs will develop. If the cancer advances even further, the GMOE could eventually develop prior to late-stage cancer. Because the genes of ancient eukaryotes have enhanced innate immunity, GMOE will eventually prevail over the weaker GMOV during cancer subgroup competition. Hence, cancer development is mainly determined by genes in the mammalian genome.

An inherent weakness of cancer cells is their dependence on glucose and iron. Furthermore, they cannot tolerate physical disturbance. Ancient gene GMOs can be treated with a combination of mechanical vibration using glucose-coated magnetic nanoparticles and strengthening of the immune system. Herein, I suggest trials for verifying this hypothesis.
\end{abstract}

Key words: Cancer origin, Viral genes, GMO, Nanoparticles 


\section{Introduction}

Attempts to weaken cancer cells prior to and during cancer treatment exploit the inherent weaknesses of cancer cells compared with normal cells. These weaknesses include dependence on glucose and iron, and weak cell structure.

\section{Glucose dependence of cancer cells}

Warburg observed that most cancer cells predominantly produce energy by a high rate of glycolysis followed by lactic acid fermentation in the cytosol. By contrast, most normal cells produce energy by a comparatively low rate of glycolysis followed by pyruvate oxidation in the mitochondria. A cancer cell requires more glucose because its metabolism is inefficient compared with normal cells. Exploiting this difference between cells, Walker-Samuel et al. developed a new technique for detecting cancer by imaging the consumption of sugar with magnetic resonance imaging (MRI) [1]. In this technique, large amounts of glucose taken up by tumors appear as bright images on the MRI scan. This method demonstrates that cancer cells absorb more glucose than normal cells. Although limiting glucose intake could reduce cancer growth, I do not advocate completely avoiding glucose intake, but rather minimizing it as much as possible.

\section{Iron is required for cancer cell proliferation}

Lam stated that "Iron is required for cell division, and it is well known that many cancer cell types selectively accumulate iron for this purpose. Most cancers have a large number of iron-attracting transferring receptors on their cell surface compared with normal cells. The Chinese herb artemisinin works via highly reactive oxygen-based free radicals that are activated in the presence of iron [2]." Hann et al. showed that tumors grow more slowly and were smaller in mice on a low iron diet than in mice on a normal iron diet [3].

\section{Mechanical vibration}

Zhang et al. designed a dynamic magnetic field generator that could induce rotational movements of superparamagnetic iron oxide nanoparticles (SPIONs). They hypothesized that the shear forces created by the generation of oscillatory torques (incomplete rotation) in SPIONs bound to lysosomal membranes would cause membrane permeabilization, leading to the extravasation of lysosomal contents into the cytoplasm and the induction of apoptosis [4]. However, because the nanoparticles were able to damage normal cells as well as cancer cells, I do not suggest the use of nanoparticles to directly kill cancer cells, but instead to only disturb them. Mechanical vibration may disturb cancer cells more than normal cells, and it is envisioned that the immune system may recognize these disturbed cancer cells.

\section{The mammalian genome and viral relics}

Up to $98 \%$ of the mammalian genome is comprised of non-coding genes, also called junk genes, which are believed to be genetic remnants which have lost most of their function during evolution. The genomes of nearly all healthy human cells contain the entirety of an individual's inherited information [5]. Not all junk genes are genetic relics of viruses, yet there are many relics of viral genes embedded in junk genes. These genes have been inactivated and have little impact on mammalian life. There is, however, evidence that such viral genes are linked to cancer.

Kozeretska et al. stated that "The mobile elements $(M E)$, also called junk DNA or transposable elements, are present in the genome of all known eukaryotes... MEs often take part in important genomic functions and provide material for natural selection, and failures and errors in their function lead to genome damage and disease, including cancer... It has become evident that the role of MEs in the initiation of some tumor types in vertebrates should be considered as an inevitable consequence of their vast genomic involvement in the generation of somatic cell diversity [6]."

Moreover, Katzourakis et al. stated that "Retroviruses have been invading mammalian genomes for over 100 million years, leaving traces known as endogenous retroviruses $(E R V s) \ldots$ with humans largely containing inactive lineages of $E R V$ s... Retroviral integration can cause cancer." The more ERV genetic relic there is in a mammalian genome, the greater the possibility that the mammal will develop cancer [7].

Kapranov et al. stated that "The function of the non-coding portion of the human genome remains one of the most important questions of our time. Its vast complexity is exemplified by the recent identification of an unusual and notable component of the transcriptome - very long intergenic non-coding RNAs, termed vlincRNAs... Finally, we show that vlincRNAs can be syntenically conserved in humans and mouse and their depletion using RNAi can cause apoptosis in cancerous cells [8]."

\section{Genetically modified organisms}

Genetic engineering is the process of manually incorporating new DNA into an organism to create one or more traits that are not already found in that organism. When a gene for a desirable (or undesirable) trait is taken from one organism and inserted into another, it gives the recipient organism the ability to express that same trait. The conventional genetic engineering approach is to insert external genetic material into the host genome. In cancer development, the concept of a genetically modified organism (GMO) implies the presence of undesirable viral genes that 
have been inactivated or silenced. If in the reproduced cell this viral gene is activated, then that active viral gene could behave like external genetic material. Thus, the new cell is a GMO, as the mutant cell and the GMO represent the same cell.

This concept of cancer as a GMO suggests that cancer developed through the incorporation of active genes into new cells. The cancer cell is a new GMO with the traits of active genes. For example, a GMO arising from the ERV gene has the traits of the ERV, whereas a GMO arising from an ancient eukaryotic gene has the traits of ancient eukaryotes.

\section{Comparing the advanced cancer cell with an- cient eukaryotes}

Table 1. The comparison of normal cells, late stage cancer cells, and ancient eukaryotes

\begin{tabular}{|c|c|c|c|}
\hline Description & Normal cell & $\begin{array}{l}\text { Advanced cancer } \\
\text { cell }\end{array}$ & $\begin{array}{l}\text { Ancient eukary- } \\
\text { ote cell }\end{array}$ \\
\hline Metabolic nutrient & Glucose, etc. & $\begin{array}{l}\text { Glucose or glu- } \\
\text { tamine }\end{array}$ & Glucose \\
\hline Metabolism & Oxidation & Toward hypoxia & Hypoxia \\
\hline Cell reproduction & Replacement only & Uncontrolled & Uncontrolled \\
\hline Iron content & Low & High & High iron \\
\hline $\begin{array}{l}\text { Radiation re- } \\
\text { sistance }\end{array}$ & Poor & Better tolerated & Better tolerated \\
\hline $\begin{array}{l}\text { Cell growth envi- } \\
\text { ronment }\end{array}$ & $\begin{array}{l}\text { Habitable envi- } \\
\text { ronment }\end{array}$ & On soft tissues & Lived in water \\
\hline
\end{tabular}

\section{Metabolism}

Similar to ancient eukaryotes, advanced cancer cells live in hypoxic environments and metabolize glucose by fermentation. Mammals with such an aerobic metabolism evolved a few hundred million years ago.

\section{Cell reproduction}

Normal cells communicate with other cells and cease to grow when they contact other cells. Cancer cells, by contrast, maintain a high proliferation rate when nutrients are available and the microenvironment is suitable.

\section{Iron content}

Before the oxygenation of the atmosphere, abundant free iron, which is required for cell division, was available on the Earth. Cancer cells require iron for their proliferation. Free iron on Earth had been oxidized prior to mammals evolving.

\section{Radiation resistance}

Until the rich oxygenation of the Earth's atmosphere, the ozone layer did not exist in the upper atmosphere. Although radiotherapy and chemotherapy function by damaging cancer cell DNA, ancient eukaryotic genes and late-stage cancer genes have resistance to high-energy impacts with the capacity to repair their damaged genetic material. This is one of the reasons cancer cells develop resistance to radiotherapy and chemotherapy. Lineweaver et al. suggest that cancer treatments should not target the cancer's strength [9].

\section{Cell growth environment}

Until the rich oxygenation of the Earth's atmosphere, the land was too harsh for living cells because no ozone layer existed to reduce the radiation from space. Tan et al. showed that cancer thrives in softer spaces and lurks quietly in stiffer cellular environments [10]. These softer spaces are similar to the aqueous environment serving as habitats for early eukaryotic cells.

The table above shows that the advanced cancers have the traits of ancient eukaryotes.

\section{Suggested trials}

\section{Suggested trial one}

The three weakening procedures described above could be combined in a protocol that uses the following equipment:

Magnetic field: A coil device could be used to generate a low frequency, uniform, alternating magnetic field to mechanically vibrate magnetic nanoparticles.

Nanoparticles: A central magnetic nanoparticle could be coated with a layer of glucose. Examples of similar sugar-coated nanoparticles have been previously explored by Dennis et al., who coated iron oxide compounds with glucose [11] and Cohen et al., who devised a cluster of nanoparticles composed of fats and coated it with a type of polysugar. When filled with chemotherapy drugs, these clusters accumulated in tumors and caused a 25-fold increase drug accumulation in tumors. A drastic decline in the toxic accumulation of drugs in healthy organs was observed [12]. Finally, Burridge demonstrated that nanoparticles can exert a mechanical force on the cells through a magnetic pulse [13]. The purpose of this trial would be to demonstrate whether the weakening procedure could aid an existing cancer treatment.

\section{Suggested trial two}

Deng et al. created a multi-layer of materials around a nanoparticle's core by creating "layer-by-layer" nanoparticles [14, 15]. This trial would be identical to trial one, except that the anti-cancer drug would be located in the middle layer of the magnetic nanoparticle. The outer layer would consist of glucose. Mechanical vibration serves a dual purpose. In addition to the physical disturbance of the cancer cells, the vibration removes the outer glucose coating, exposing the cancer cell to the anticancer drug. Me- 
chanical vibration would be the method of delivering the anticancer drug into the cancer cell. The challenge would be to develop an anticancer drug that has minimum impact on normal cells.

Duke University (NC, USA) researchers found that high-intensity focused ultrasound shakes cancer cells. This effect, which was observed in mice, may also apply to human patients [16]. However, the ultrasonic wave vibration exerts the same force on both cancer and normal cells. Therefore, compared with ultrasonic vibration, the combination of glucose-coated nanoparticles with mechanical vibration would have a greater impact on cancer cells.

\section{Application of glucose-coated nanoparticles}

I suggest the use of glucose-coated nanoparticles as a cancer treatment because it does not rely on novel, breakthrough technology, and the majority of research institutes would be able to carry out the suggested trials. The magnetic coil design should generate a uniform magnetic field where the cancer cells are located.

If the alternative magnetic field strength is uneven, cells may be subjected to stronger parts of the magnetic field. Hence, there is a risk of harming normal cells. A rotational magnetic field [4] may not be suitable for this application because it has a constant angular velocity with a linear speed varying from the distance to the center.

The glucose-coated nanoparticles would more readily enter cancer cells than normal cells because cancer cells have a greater requirement for glucose [1]. The vibration would then disturb and weaken the late-stage cancer cell. This hypothesis could be easily validated by trials.

\section{Hypotheses regarding the origin of cancer}

I hypothesize that:

A. Ancient eukaryotes existed prior to the presence of free oxygen on the Earth and metabolized glucose under hypoxic conditions.

B. After the rich oxygenation of the Earth's atmosphere, multi-cellular development formed diverse and functional cells and differentiated cells with oxidative metabolisms.

C. Many genes have been exchanged by HGT throughout cellular evolution. Most of the mammalian genome is comprised of non-coding genes, or junk genes, which are evolutionary remnants.

D. Although the majority of viral genes are inactive during the course of human life, some may, on occasion, become active and be involved in the development of the placenta when required.

E. Mammals may undergo millions of cell regenerations in their daily life, but not all reproduced cells can be normal and healthy. If the process of cell reproduction is defective or mutated, there are several potential consequences for the new cell.

1. The damaged DNA must be repaired. If this is not possible, then

2. The immune system eradicates the mutant cell. If not, then

3. The mutant cells develop into a benign tumor if the viral genes remain inactive, or

4. If the viral genes of the new cell are actively involved, which is similar to genetic engineering, the new cell is transformed into a GMO by that viral gene.

5. The mammalian genome consists of many relics of viral genes, and it is unknown whether these genes are harmful. Various types of GMOs could be created depending on which gene becomes active. Cancer cells are amalgamations of various groups of GMOs.

6. The new GMO may no longer be able to inactivate the viral genes or the deeply rooted genes of eukaryotes. Other inactive genes could become active, subsequently creating another new GMO. In this way, cancer cells continuously create more GMOs as the cancer progresses.

F. The physical structure of cancer cells is fragile in comparison with normal cells, which can be rationalized as follows:

- The mammalian cell evolved over millions of years, whereas the development of cancer cells occurs under uncontrolled proliferation. In addition, cancer blood vessels are leaky. Finally, normal cells are optimized for stability, whereas cancer cells are optimized for growth.

- Ultrasonic mechanical vibration has a greater impact on cancer cells than on normal cells [16].

- In mouse models, fasting prior to chemotherapy ensures cancer treatment is $40 \%$ more effective with no noticeable effect on normal cells [17].

- Late-stage cancer cells have characteristics similar to ancient eukaryotes. All living organisms have finite resources. In order to survive in the harsh environment billions of years ago, a crucial survival requirement for a eukaryote was to have a strong cell membrane. Hence, eukaryotes devoted less of their resources to internal defense structures.

- Physical weakness is common in late-stage cancer cells. This weakness should be targeted by physically disturbing the cancer cell.

\section{Theory of cancer origin}

Cancer can be induced by external viruses, such as papillomaviruses. Cancer can also be induced by other external agents, such as radiation and chemical 
carcinogens [18]. However, the origin of cancer is not external agents, but the mammalian genome. There are two existing theories of cancer origin, which are based on cellular malfunction.

\section{The Somatic Mutation Theory}

"The premises of this theory are as follows: (1) cancer is derived from a single somatic cell that has accumulated multiple DNA mutations; (2) the default state of cell proliferation in metazoa is quiescence; and (3) cancer is a disease of cell proliferation caused by mutations in genes that control proliferation and the cell cycle [19]."

In GMO hypotheses, although external agents can induce GMO development in many instances, external agents are not necessary for all GMO development. Most new GMO developments are random events. The cancer cell, GMO, with viral traits, has mutational capability. If such a mutation does occur, it only plays a minor part in cancer development.

\section{Cancer as a Metabolic Disease Theory}

Another cancer origin theory stated that "Any unspecific condition that damages a cell's respiratory capacity, but is not severe enough to kill the cell, can potentially initiate the path to a malignant cancer," and "cancer is not a genetic disease but a metabolic disease involving mitochondrial dysfunction and respiratory insufficiency [20]." Scientists have not yet been able to demonstrate that metabolic disease is the cause rather than the consequence of cancer development.

\section{Cancer has a deep evolutionary root}

Davies et al. hypothesized that "cancer is a type of throwback, or atavism, to an ancestral phenotype. Cells are usually regulated by mechanisms that instruct them when to multiply and when to die. What we believe is that when these mechanisms malfunction, the cells revert to the default option, a genetic subroutine programmed into their ancestors long ago, of behaving in a selfish way. To use a computer analogy, cancer is like Windows defaulting to 'safe mode' after suffering an insult of some sort [5]." In other words, cancer is driven by genes deeply rooted in the tree of multi-cellular life. The GMO hypothesis for cancer has a similar concept; it is the ancient genes that ultimately drive cancer development but with a different interpretation.

In the GMO hypotheses, cancer develops when the relics of viral genes are incorporated into new cells in the cell cycle, similar to genetic engineering. The new GMO cell expresses the traits of the viruses from which the relics of viral genes arose. The GMO may contain more than one viral gene relic and the GMO will express traits of all viral characteristics. Cancer development could have multiple types of GMO, similar to genetic engineering using various viral genes. Therefore, cancer is an amalgamation of
GMOs.

GMOs comprise two main groups. One group arises by genetic engineering of viral gene relics in the junk genes, which evolved after the oxygenation of the atmosphere (GMOV). The GMOV express the traits of viruses that evolved during the last half billion years (the Phanerozoic period). The other arises by genetic engineering of genes from early eukaryotes that existed prior to the oxygenation of the Earth (GMOE). These genes are deeply embedded in the genome (the Proterozoic period, $0.5-2.5$ billion years ago) with aggressive proliferation and good survival capability.

The hypothesis also states that most cancers have their origins in GMOV. As the cancer progresses, further subgroups of cancer GMOs will develop. If the cancer advances further, the GMOE could eventually develop prior to late-stage cancer.

Polyak et al. observed that "individual tumors are genetically heterogeneous - comprised of multiple subgroups of cancer cells, each with its own genomic signature or pattern of gene mutations... and when multiple subgroups were present in the same tumor, they interfered with each other's expansion [21, 22]." The observation that multiple subgroups of cancer cells compete with each other would support amalgamated GMOs hypotheses because multiple GMOs compete with each other in cancer development.

Earlier organisms had superior innate immunity and poor adaptive immunity. As evolution progressed, organisms exhibited superior adaptive immunity and poorer innate immunity [9]. Basler et al. observed that "In two genetically distinct contexts of cell competition, the ancient innate immune defense response system is activated and drives the elimination of the cells perceived as relatively less fit [23]," and so the stronger ancient innate immune GMOE will eventually prevail over the weaker GMOV during cancer development. These two observations support the interpretation of GMO hypotheses.

\section{Can "Cancer cells as an amalgamation of GMOs" hypotheses be validated?}

Heidmann et al. reconstructed the DNA sequence of a retrovirus and showed that it is able to produce infectious particles, the retrovirus named Phoenix [24]. If, in an experiment, a normal cell could be genetically engineered by a viral gene (from a junk gene) to create a cancerous GMO, this would validate the GMO hypotheses.

\section{Suggested treatment strategies}

When two opposing entities compete, one will prevail if it is stronger or its opponent is weaker.

Ben-Jacob et al. observed that the best strategy to 
fight cancer is to strengthen the immune system and simultaneously weaken the cancer [25].

\section{Improving immunity}

Anderson applied the Lymphocyte Genome Sensitivity test, which measures the damage caused to the DNA of white blood cells that are under stress while fighting cancer, to find that individuals with cancer have DNA that is more easily damaged by ultraviolet light than those without cancer [26].

This result shows that white blood cells are under stress in cancer patients. Hence, the immune systems of cancer patients should be improved. We should adapt the best available technology to strengthen the immune system.

\section{Weakening the cancer GMO}

Glucose-coated nanoparticles may not be affected by the mammalian immune system, and thus would have a strong likelihood of entering cancer cells. If glucose-coated nanoparticles could successfully enter and disturb cancer cells, the following treatments could be realized:

1. Cancer is easier to treat if detected early by improved diagnostic methods. The relics of viral genes are embedded in the junk genes, such as ERV, which evolved after the increase in free oxygen on the Earth. Most cancers have their origins in GMOV. This type of GMO is less resistant to DNA damage and would not be as aggressive in its proliferation as GMOE. The anticancer drugs in suggested trial two could then be used for treatment.

2. GMO genes may have arisen from the eukaryotes that evolved prior to the rich oxygenation of the Earth's atmosphere. The GMOE proliferates when in its favorable environment, otherwise it will remain dormant. This comfortable environment can be disrupted by reducing glucose levels and iron intake to retard cancer proliferation, GMOE, without killing it. The GMOE could be dormant for a long period.

3. As the GMOE derived from ancient genes will eventually drive the development of cancer, it is possible to exploit the weaker structure of cancer cells and the lack of adaptive immunity in GMOE [9]. Mechanical vibration would agitate the GMOE cells and create a disturbance to which the GMOE could not respond. This approach would give the immune system a chance to attack the cancer cells.

4 . The same procedures could be used in treating or eradicating circulating cancer cells in blood vessels.

Because the ancient eukaryotes lived in anaerobic environments, it is possible that hyperbaric oxygen therapy may weaken the GMOE. More studies may be required.

\section{Discussion}

There are three hypotheses in this manuscript as follows: (1) by glucose-coated nanoparticle vibration, late-stage cancer cells suffer a greater weakening effect than normal cells; (2) cancer develops if active viral genes are incorporated into new cells, GMOV, in the cell cycle, similar to genetic engineering. Both these hypotheses could potentially be verified by laboratory experiments; and (3) GMOE will eventually drive cancer development if cancer progresses further.

The environment on Earth evolved from an almost non-free oxygen environment to an environment rich in oxygen approximately half a billion years ago; hence, life evolved from anaerobic to aerobic metabolism. The majority of the mammalian genome is represented by junk genes, which are remnants of evolutionary history. These viral relics have been inactivated through evolution, and many reports have stated that some viral genes are linked to cancer $[6,7$, 8]. Davies stated that "Lineweaver and I envisage cancer progression within a host organism as like running the arrow of biological evolution backward in time at high speed...cancer generally cannot be cured [5]". In GMO hypotheses, by hypothesizing the late-stage cancer is GMOE, there is a possibility to develop cancer treatment strategies.

By reverse engineering, it has been shown that the more prevalent the ERV in a mammalian genome, the greater the possibility that the mammal will develop cancer [7]. In addition, it has been observed that late-stage cancers have the same characteristics as ancient eukaryotes. These two observations imply that cancer development is primarily determined by genes in the mammalian genome.

\section{Abbreviations}

HGT: horizontal gene transfer; GMO: genetically modified organism; ERV: endogenous retroviruses; GMOV: GMO arising from genetic relics of a virus that evolved after oxygenation of the Earth; GMOE: GMO arising from genes of eukaryotes that evolved before the oxygenation of the Earth.

\section{Competing Interests}

The author has declared that no competing interest exists.

\section{References}

1. Walker-Samuel S, Ramasawmy R, Torrealdea F, Rega M, Rajkumar V, Johnson SP, Richardson S, Gonçalves M, Parkes HG, et al. In vivo imaging of glucose uptake and metabolism in tumors. Nature Medicine. 2013; 19: 1067-72

2. [Internet] Lam M. Artemisinin (Wormwood) - From Malaria to Cancer. http:/ / www.drlam.com/articles/Artemisinin.asp?page=1

3. Hie-Won L, et al. Iron Nutrition and Tumor Growth: Decreased Tumor Growth in Iron-deficient Mice. Cancer Res. 1988; 48: 4168-70 
4. Zhang E, Kircher MF, Koch M, Eliasson L, Goldberg SN, Renström E. Dynamic Magnetic Fields Remote-Control Apoptosis via Nanoparticle Rotation. ACS Nano. 2014; 8 (4): 3192-201

5. [Internet] Davies P. Exposing cancer's deep evolutionary roots; Physics World; July 1, 2013. http://physicsworld.com/cws/article/indepth/2013/ jul/01/exposing-cancers-deep-evolutionary-roots

6. Kozeretska IA, Demydov SV, Ostapchenko LI, Shevchenko T. Mobile genetic elements and cancer. From mutations to gene therapy. Experimental Oncology. 2011; 33 (4): 198-205

7. Katzourakis A, Magiorkinis G, Lim AG, Gupta S, Belshaw R, Gifford R, et al Larger Mammalian Body Size Leads to Lower Retroviral Activity. PLoS Pathogens, 2014; 10 (7): e1004214

8. St Laurent G, Shtokalo D, Dong B, Tackett MR, Fan X, Lazorthes S, Nicolas E, Sang N, Triche TJ, McCaffrey TA, Xiao W, Kapranov P. VlincRNAs controlled by retroviral elements are a hallmark of pluripotency and cancer. Genome Biology. 2013; 14 (7): R73

9. Lineweaver $\mathrm{CH}$, Davies PCW, Vincent MD. Targeting cancer's weaknesses (not its strengths): Therapeutic strategies suggested by the atavistic model. BioEssays. 2014; 36 (9): 827-35

10. Tan Y, Tajik A, Chen J, Jia Q, Chowdhury F, Wang L, Chen J, Zhang S, Hong Y, Yi H, Wu DC, Zhang Y, Wei F, Poh Y, Seong J, Singh R, Lin L, Doğanay S, Li Y, Jia H, Ha T, Wang Y, Huang B, Wang N. Matrix softness regulates plasticity of tumour-repopulating cells via H3K9 demethylation and Sox2 expression. Nat Commun. 2014; 5: 4619

11. Dennis CL, Jackson AJ, Borchers JA, Hoopes PJ, Strawbridge R, Foreman AR, van Lierop J, Gruttner C, Ivkov R. Nearly complete regression of tumors via collective behavior of magnetic nanoparticles in hyperthermia. Nanotechnology. 2009; 20 (39): 5103

12. Cohen K, Emmanuel R, Kisin-Finfer E, Shabat D, Peer D. Modulation of Drug Resistance in Ovarian Adenocarcinoma Using Chemotherapy Entrapped in Hyaluronan-Grafted Nanoparticle Clusters. ACS Nano. 2014; 8 (3): 2183-95

13. Lessey-Morillon EC, Osborne LD, Monaghan-Benson E, Guilluy C, O'Brien ET, Superfine R, Burridge K. The RhoA Guanine Nucleotide Exchange Factor, LARG, Mediates ICAM-1-Dependent Mechanotransduction in Endothelial Cells To Stimulate Transendothelial Migration. The Journal of Immunology. 2014; 192 (7): 3390-98

14. Deng ZJ, Morton SW, Ben-Akiva E, Dreaden EC, Shopsowitz KE, Hammond PT. Layer-by-Layer Nanoparticles for Systemic Codelivery of an Anticancer Drug and siRNA for Potential Triple-Negative Breast Cancer Treatment. ACS Nano. 2013; 7 (11): 9571-84

15. Tang $L$, Yang $X$, Yin $\mathrm{O}$, Cai $\mathrm{K}$, Wang $\mathrm{H}$, Chaudhury I, Yao C, Zhou O, Kwon M, Hartman JA, Dobrucki IT, Dobrucki LW, Borst LB, Lezmi S, Helferich WG, Ferguson AL, Fan TM, Cheng J. Investigating the optimal size of anticancer nanomedicine. Pnas. 2014; 111 (43):15344-49

16. [Internet] Duke University, High-Intensity Ultrasound May Launch Attack On Cancer, Wherever It Lurks. ScienceDaily, 8 August 2007. www.sciencedaily.com/releases/2007/08/070807101114.htm

17. [Internet] Harmon K. Fasting Might Boost Chemo's Cancer-Busting Properties; Scientific American; Feb 8, 2012. http://www.scientificamerican.com/ article.cfm?id=fasting-might-boost-chemo

18. Cooper GM, Hausman RE. The Cell: A molecular Approach; 2nd edition. Sinauer Associates; 2000

19. Soto AM, Sonnenschein C. The somatic mutation theory of cancer: growing problems with the paradigm? Bioessays. 2004; 26 (10): 1097-107

20. Seyfried T. Cancer as a Metabolic Disease. Wiley. 2012

21. Marusyk A, Tabassum DP, Altrock PM, Almendro V, Michor F, Polyak K. Non-cell-autonomous driving of tumour growth supports sub-clonal heterogeneity. Nature. 2014; 514: 54-58

22. [Internet] Dana-Farber Cancer Institute. Target growth-driving cells within tumors, not fastest-proliferating cells; ScienceDaily, 30 July 2014. www.sciencedaily.com/releases/2014/07/140730133133.htm

23. Meyer N, Amoyel M, Bergantinos C, de la Cova C, Schertel C, Basler K, Johnston LA. An ancient defense system eliminates unfit cells from developing tissues during cell competition. Science. 2014; 346 (6214): 1258236

24. [Internet] Dewannieux M, Harper F, Richaud A, et al. Identification of an infectious progenitor for the multiple-copy HERV-K human endogenous retroelements. http://genome.cshlp.org/content/early/2006/10/31/ gr.5565706.full.pdf $+\mathrm{html}$

25. Lu, Huang B, Hanash SM, Onuchic JN, Ben-Jacob E. Modeling putative therapeutic implications of exosome exchange between tumor and immune cells. Pnas. 2014; 111 (40): 4165-74

26. Anderson D, Najafzadeh $M$, Gopalan $R$, Ghaderi N, Scally AJ, Britland ST, Jacobs BK, Reynolds PD, Davies J, Wright AL, Al-Ghazal S, Sharpe D, Denyer MC. Sensitivity and specificity of the empirical lymphocyte genome sensitivity (LGS) assay: implications for improving cancer diagnostics. The FASEB Journal. 2014; 28 (10): 4563-67 